\title{
Der Kunde und Doc Google - wie können wir konstruktiv mit Netzdiagnosen umgehen?
}

\author{
Antje Blättner
}

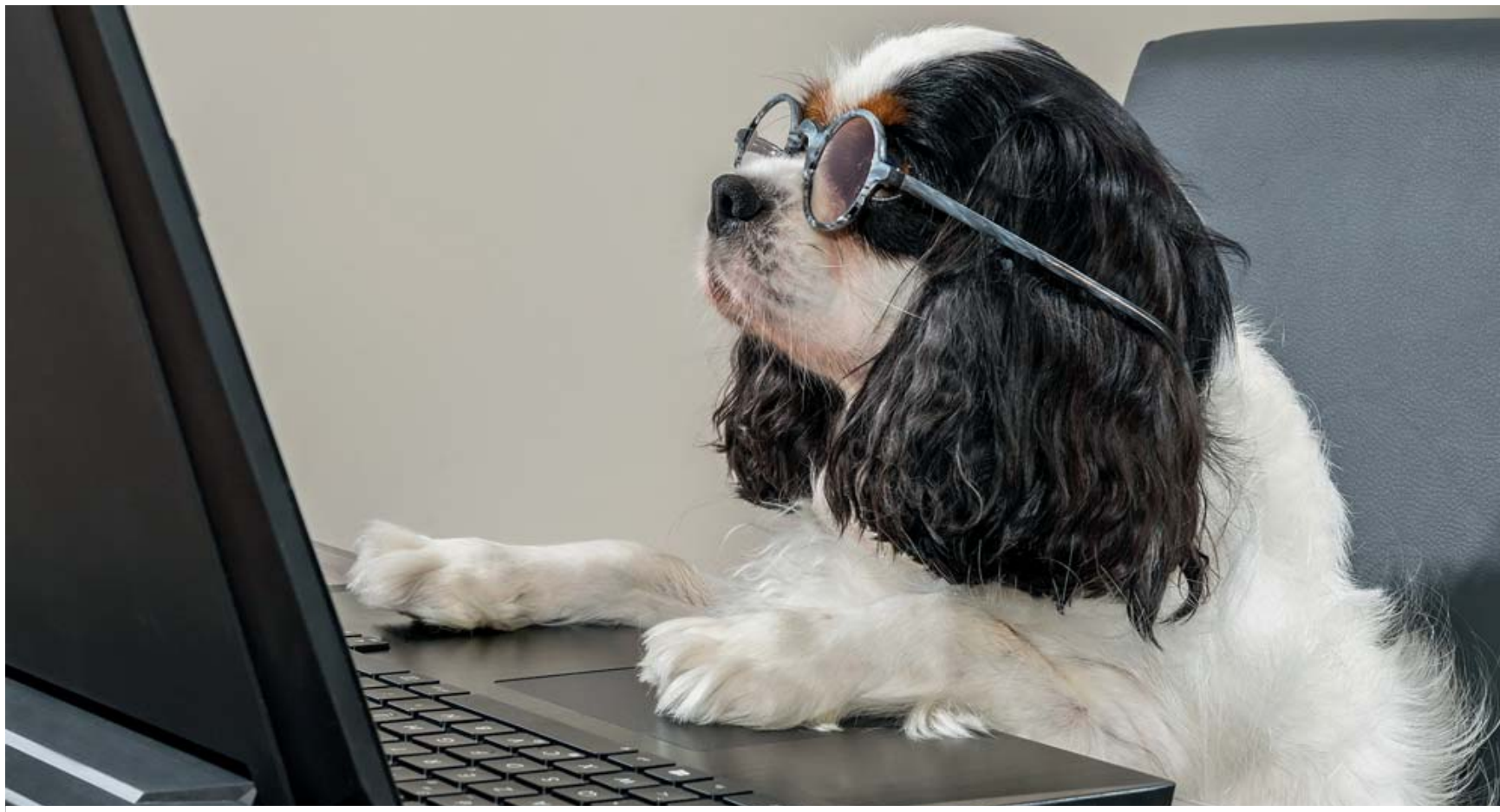

(c) Pictures news - stock.adobe.com

\begin{abstract}
Alle sind im Netz unterwegs, so auch Ihre Kunden. Und sie finden dort Antworten auf ihre Fragen, zu denen sie nicht immer passende Antworten vom Praxisteam bekommen. Wir zeigen, wie Sie mit guter Zeitplanung Kundengespräche in die Hand nehmen und in die richtige Bahn lenken können.
\end{abstract}

Gesundheitscheck mit oder ohne Impfung - das ist fast immer eine Routinehandlung für viele Praxisteams und stellt im Praxisalltag eine (meist) gut planbare und vorhersehbare Konsultation dar. Doch was ist, wenn der Kunde überraschend seinen „Netzfund“ zum Thema Parasitenkontrolle mitbringt und diesen diskutieren möchte? Dann wird es spannend - im wahrsten Sinn des Wortes!

\section{Die Ausgangslage}

Der erste „Fehler“ bei der Planung eines Gesundheitschecks ist oft, dass es keine Planung gibt, und das generiert Spannung und Zeitdruck. Das bedeutet: Selten setzt sich das Team zusammen und überlegt, was eigentlich genau zum Leistungsumfang bei einem Gesundheitscheck in puncto Leistung, Beratung und Produkten ge- hört. Man geht meist davon aus, dass „Impfung“ einfach ist: körperliche Untersuchung, evtl. Befunde besprechen, Pieks, fertig. Mit diesem Regime ist die Gefahr natürlich groß, dass 1-2 (kritische) Fragen oder Beratungswünsche des Kunden die „Zeitplanung“ komplett aus dem Ruder laufen lassen, zumal viele Praxismanagement-Systeme im Auslieferungszustand einen Terminrhythmus mit 15 min vorgeben, der dann brav (aber oft gedankenlos) befüllt wird. So entsteht zunächst hausgemachter Zeitdruck, der dann im Praxisalltag zu Verzögerung und Stress bei Team und Kunden führt.

\section{Stressfreie Planung}

Eine Zeiteinteilung für Routineverrichtungen wie z. B. Gesundheitschecks, Kontrolluntersuchungen oder Durchfallbehandlungen kann und sollte grundsätzlich so ge- 


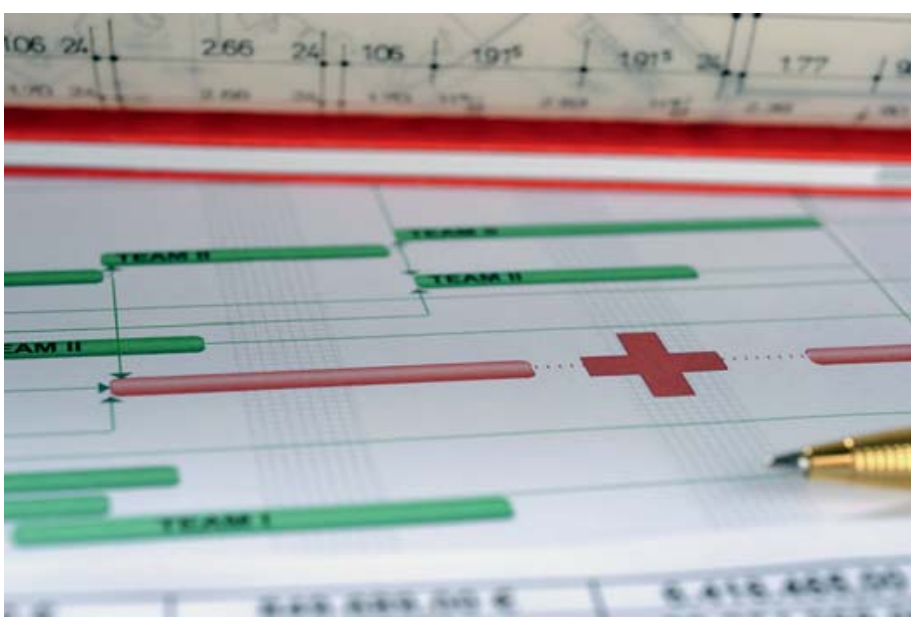

- Abb. 1 Eine gute Terminplanung am Telefon beruht auf der realistischen Einschätzung des Zeitbedarfs für eine Konsultation mittels Zeitraster plus bestimmten Fragen an den Tierhalter. @ CHW - stock.adobe.com

plant werden, dass immer Zeit bleibt, auf Eventualitäten oder „Sonderwünsche“ des Kunden reagieren zu können. Und hier ist natürlich die Terminsprechstunde haushoch überlegen, d. h. der Zeitbedarf für eine Konsultation kann im Vorfeld wesentlich besser eingeschätzt und damit auch besser und realistisch in die zur Verfügung stehenden Arbeits- bzw. Öffnungszeiten der Praxis integriert werden. Eine reine Terminpraxis hat zudem den Vorteil, dass das Team sich wesentlich besser auf den Kunden vorbereiten und ihn und sein Tier individueller und auch qualitativ besser versorgen kann, als wenn man nacheinander alles „abarbeitet“, was sich ins Wartezimmer gesetzt hat: „schnelle“ Impfungen, anspruchsvolle dermatologische Aufarbeitungen, Kontrolluntersuchungen und auch Notfälle (echte und die, die der Kunde als solche definiert und Druck macht). Es wird klar: Das kann nicht wirklich gut funktionieren - irgendwer bleibt immer auf der Strecke.

\section{Zeitraster erstellen}

Natürlich lässt sich das dynamische Geschehen einer Tierarztpraxis nicht minutiös Tag für Tag planen und in ein starres Korsett zwingen. Wenn aber folgende Punkte Anwendung finden, kann die Zeitplanung tatsächlich deutlich entstresst werden:

- Auflistung der häufigsten Routinekonsultationen, z. B. Gesundheitscheck (mit/ohne Impfung).

- Festlegung der Leistungen und Produkte für den Gesundheitscheck, z.B. vollständige körperliche Untersuchung mit Erläuterung der Befunde und Empfehlungen für Gesundheitsvorsorge inkl. Ekto- und Endoparasitenkontrolle und Ernährung.

- Definition des Zeitbedarfs für die Konsultation mit den festgelegten Inhalten.

Erweiterung dieses Zeitbedarfs um 5-10 min, um einen Zeitpuffer für Eventualitäten zu schaffen.
Mit dieser Vorgehensweise lässt sich nun Schritt für Schritt ein Zeitraster für die häufigsten Konsultationen erstellen, das - zusammen mit einer sorgfältigen Planung am Telefon - als Basis für die tägliche Terminvergabe dient. Das Zeitraster sollte im PC und als Ausdruck am Telefonplatz (Rezeption) hinterlegt sein, sodass jedes Teammitglied jederzeit Zugriff hat ( $\bullet$ Abb. 1).

Dieses Konzept empfiehlt sich immer, auch wenn die Praxis (noch) keine reine Terminsprechstunde führt. Denn auch dann ist es sinnvoll, dass das Team eine einheitliche Vorgehensweise bei bestimmten Konsultationen hat und damit die Leistung und Qualität für alle Kunden die gleiche ist (Stichwort QM).

Und sollte mal wirklich „zu viel“ Zeit übrig sein, dann gibt's eben einen Kaffee in Ruhe, werden Laborberichte gelesen, Recalls erledigt, Personalgespräche geplant, das Protokoll der letzten Teamsitzung aufgearbeitet, die Tagespost erledigt, die nächste Fortbildung ausgewählt oder ein Fachartikel gelesen... Dinge, die immer wieder im Alltag untergehen.

Merke

„Frei-Zeit" füllt sich meist von ganz allein, und wenn nicht: Entspannung tut auch gut!

\section{Termine am Telefon}

Damit eine gute Zeitplanung etabliert werden kann, muss natürlich auch der Kunde am Telefon miteinbezogen werden, denn auch heute geht dem persönlichen Kontakt meist ein telefonischer voraus, und diese Chance sollte genutzt werden. Das bedeutet: Die TFA am Telefon soll beim Anruf des Kunden den Konsultationsgrund erfragen, dabei jedoch weitere, für ein gutes Zeitmanagement wichtige Details erfassen - d.h., von sich aus aktiv ansprechen und wie in einer Checkliste abarbeiten:

- Welche weiteren Konsultationswünsche bestehen für das angesprochene Tier? Kunden „vergessen“ ab und an, was noch außer der Impfung erledigt werden soll, so z.B. Krallen schneiden, Analbeutel kontrollieren und entleeren und (beide) Ohren untersuchen. Das kommt dann in der Praxis zur Sprache, und der Tierarzt muss entscheiden: Alles in den engen Zeit-Slot von 15 min reinquetschen oder auf 2 Konsultationen verteilen? Meist entscheiden sich Kollegen für das Zeitraffer-Programm mit der Folge, dass andere wichtige Themen nicht angesprochen werden und unter Druck schnell gearbeitet wird.

- Mit wie vielen Tieren kommt der Kunde? Oft vereinbaren Kunden für 1 Tier einen Termin, bringen dann jedoch noch 1 Tier mit (oder noch mehr), weil es gerade praktisch erscheint. Und natürlich denkt der Kunde nicht daran, wie es die Zeiteinteilung der Praxis über den Haufen wirft, das ist ja auch nicht sein Job.

- Welche Konsultationswünsche bestehen für die weiteren Tiere? 
- Zu welchen zusätzlichen Themen möchte der Kunde eine Beratung? Auch ein Dialog über das pro und kontra einer Kastration kann locker mehr als 10 min in Anspruch nehmen - open end, je nach Kunde. Und dann sitzt der Tierarzt auf glühenden Kohlen, weil alle nachfolgenden Kunden im Wartezimmer mit den Hufen scharren. Und in dieser Situation ist eine sorgfältige und überzeugende Beratung nicht mehr gut zu leisten.

Wichtig ist dabei, diese Fragen aktiv zu stellen und sich nicht darauf zu verlassen, dass der Kunde von sich aus mehrere Tiere anmeldet, und das jeweils mit einem oder mehreren präzise formulierten Konsultationswünschen und mit den Themen, über die er beraten werden möchte.

Wenn am Telefon jedoch alle Fragen gestellt und alle Antworten erfasst wurden, kann zusammen mit dem oben angesprochenen Zeitraster eine realistische Zeit für die individuelle Konsultation eingeplant werden - und die ist dann eben nicht 15, sondern eher 30-40 min. Und diese Konsultation sollte dann natürlich auch mehr kosten, als eine 15-minütige, finale Otitiskontrolle.

\section{Im Gespräch}

Wenn nun der Zeitbedarf für einen Gesundheitscheck mit Impfung gut geplant und am Telefon kommuniziert wurde, dann sollte genügend Spielraum vorhanden sein, um flexibel auf unerwartete Fragestellungen in der Konsultation zu reagieren, ohne dass der gesamte Zeitplan aus den Fugen gerät.

Dieser Fall kann z. B. eintreten, wenn der Kunde bei der Besprechung der Parasitenprophylaxe im Rahmen des Gesundheitschecks sagt: „Ektoparasiten? Da wollte ich für Bodo eine Bernsteinkette kaufen. Das habe ich im Netz gefunden. Ist ganz ohne Chemie und sanft." Jetzt wird es spannend, denn die Situation erfordert Fingerspitzengefühl, wenn man die Kundenfrage nicht pauschal mit „Ach, was da im Netz steht, das ist alles Quatsch, das sollte man nicht glauben!“ abbügeln will. Besser geht das z. B. so:

Kunde: Ektoparasiten? Da wollte ich für Bodo eine Bernsteinkette kaufen.

TierärztIn: Das ist ja interessant! Was steht denn da zur Wirksamkeit, gibt es eine Studie, die zeigt, wie zuverlässig Bernstein gegen Flöhe und Zecken hilft?

Kunde: Da habe ich nicht darauf geachtet. Das klang alles so sinnvoll und logisch, und man konnte die Kette dort auch gleich kaufen!

TierärztIn: Das verstehe ich! Frau Schmidt, können Sie mir denn die Informationen besorgen, damit ich sie mir an- sehen kann? Dann können wir sehen, welche Fakten es zum Bernstein gibt und sie beurteilen. Wir wollen ja nicht, dass Bodo Flöhe und Zecken bekommt oder dass Sie Geld ausgeben für etwas, das nicht wirkt, oder?

Kunde: Das stimmt, aber die chemischen Mittel sind schon echte Hämmer, oder?

TierärztIn: Wie hat Bodo denn letztes Mal auf XY reagiert?

Kunde: Gar nicht. Ich habe nichts gemerkt, aber im Netz steht, dass es Spätschäden gibt.

Tierärztln: Ich kann gut verstehen, dass Sie ein wirksames und gut verträgliches Mittel für Bodo möchten. Wenn Bodo XY gut vertragen hat, dann ist die Wahrscheinlichkeit, dass Schäden entstehen, äußerst gering. Und wenn er es nicht mehr verträgt, dann gibt es genügend Alternativen. Wir haben in unserer Praxis wirksame und gut verträgliche Präparate ausgewählt, die wir Ihnen empfehlen. Wir wollen ganz sicher nicht, dass unsere Kunden über Nebenwirkungen klagen und dass unsere Patienten Medikamente bekommen, die nicht gut vertragen werden.

Kunde: Das ist klar.

Tierärztln: Ich schlage vor, wir versorgen Bodo jetzt noch einmal mit dem Floh- und Zeckenmittel, das er gut vertragen hat. Und Sie besorgen die Infos zur Bernsteinkette und melden sich bei mir, dann sprechen wir darüber, wenn die Fakten auf dem Tisch sind. Wie klingt das?

Kunde: Das ist OK, das machen wir. Und danke, dass Sie meine Frage ernst genommen haben. Der andere Tierarzt, bei dem ich neulich im Notdienst war, der hat gesagt, dass alles, was im Internet steht, Blödsinn ist und hat mir nicht zugehört. Dann habe ich halt noch ein bisschen mehr gegoogelt, um Infos zu finden.

\section{Kernpunkte im Dialog}

Der obige Dialog kann als Muster dienen für Kundenanfragen, die entweder merkwürdig, provozierend oder „anstrengend“ erscheinen. Dabei ist es wichtig zu bedenken, dass der Kunde (meist) nicht absichtlich anstrengend ist, sondern dass er „seine“ Fachleute fragen will. Das ist gut so, denn ein Kunde, der fragt, legt immerhin (noch) Wert auf unsere Meinung und wir können in einen konstruktiven Dialog einsteigen. Der Kunde lernt dann, dass es bei seinem Praxisteam wirklich keine „dummen Fragen“ gibt und er ernst genommen wird. Dass die Infos, die Kunden im Netz finden, nicht immer gängige tiermedizinische Behandlungsstrategien widerspiegeln, liegt in der Natur der Sache. Dass wir uns damit auseinandersetzen müssen, auch. 


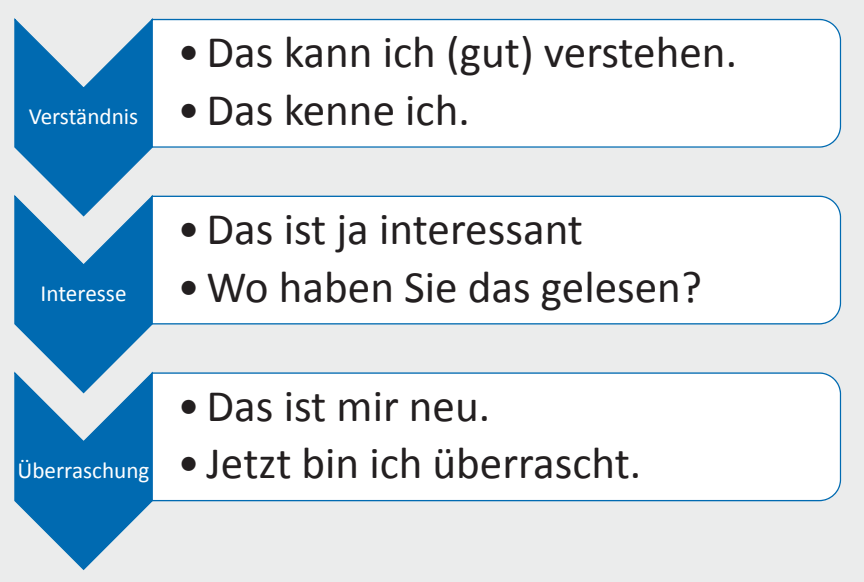

- Abb. 2 Es gibt 3 Möglichkeiten für die erste Reaktion auf eine kontroverse, merkwürdige oder provozierende Kundenanfrage. Das Ziel ist, dem Kunden Aufmerksamkeit zu signalisieren, dann das Gespräch zu übernehmen und mit Fragen weiter zu führen. @ A. Blättner

Zusammengefasst sind folgende Kernpunkte im Dialog um kontroverse Wünsche und Anfragen wichtig für den Erfolg:

Kernpunkt 1: Bei Konfrontation mit merkwürdigen oder unliebsamen Äußerungen ist es wichtig, nicht sofort mit Fachwissen und Argumenten dagegen zu schießen, denn dann wird der Dialog konfrontativ. Besser ist es, Überraschung, Interesse und Verständnis zu äußern ( $>$ Abb. 2).

Kernpunkt 2: „Wer fragt, der führt“ ist eines der Grundsätze in der Kommunikation. Es gilt also zu fragen, dabei mehr zu erfahren und den Kunden sanft auf Defizite oder Fehler beim Netzangebot aufmerksam zu machen. Fragen zu stellen, bedeutet, die Richtung des Dialogs zu bestimmen, denn nun wird der Kunde reaktiv und der Tierarzt aktiv - der Spieß ist umgedreht.

Kernpunkt 3: Man muss nicht auf alles eingehen, was der Kunde sagt. Im obigen Beispiel wird auf die Frage, ob Chemie ein echter Hammer ist, keine Antwort geliefert. Stattdessen wird erneut gefragt, und das auf dem Hintergrund einer gut geführten Kundenkartei. Diese zeigt nämlich, dass Bodo seit Jahren $4 \mathrm{x}$ im Jahr XY bekommt und noch nie eine negative Reaktion erfolgt ist.

Kernpunkt 4: Verknüpfung eines passenden Angebotes mit dem Nutzen für den Kunden - Bodo bekommt ein zuverlässiges, in der Praxis bewährtes Medikament, das er gut verträgt, und ist vor Flöhen und Zecken geschützt.

Merke

Die überzeugende Strategie in der Kommunikation ist: Mit dem Kunden, nicht gegen ihn!

\section{Google-Prophylaxe}

Natürlich können wir nicht verhindern, dass unsere Kunden im Netz auch nach Gesundheitsthemen für ihre Vierbeiner suchen. Was wir jedoch tun können, ist dafür zu sorgen, dass der Kunde in der Praxis optimal mit Informationen versorgt wird. Das bedeutet auch, Themen anzusprechen, die der Kunde nicht von sich aus explizit erfragt. Denn wenn wir warten, bis der Kunde Fragen stellt, kann es bereits zu spät sein, $d$. h. er hat sich schon schlau gegoogelt. Und: Wer kein Experte ist, kann natürlich auch nicht immer die richtigen Fragen stellen.

Ziel muss es sein, dass unsere Kunden möglichst zuerst aus ihrer Tierarztpraxis über wichtige Themen zur Tiergesundheit und Krankheitsprophylaxe informiert werden, bevor es ein anderer erledigt und wir dann korrigieren dürfen. Das bedeutet für die Praxis: Themen festzulegen, zu denen jeder Kunde proaktiv angesprochen werden soll, und sich zusätzlich Gedanken zu machen, welche Materialien für welche Themen eingesetzt werden. Dies gilt natürlich in der Regel für eine ganz „normale" Haustierarztpraxis, nicht für hochspezialisierte Einrichtungen - ein radiologisches Zentrum muss sich nicht mit Parasitenprophylaxe beschäftigen.

\section{Materialien}

Um den Kunden gut zu informieren, sollte jede Praxis für die jeweils wichtigen Themen entsprechende Materialien zur Verwendung im Dialog und zur Abgabe an den Kunden bereithalten. Die Industrie hat jede Menge Tierhalterbroschüren für alle möglichen Themen, es gilt hier: Material einsetzen, statt es als Wartezimmerdeko auszulegen: Eine Broschüre soll im Kundengespräch Verwendung finden, wichtige Inhalte sollten angestrichen werden und das Ganze vor der Abgabe mit dem Tiernamen versehen sein - das erhöht die Chance, dass das Material es bis nach Hause schafft und auch noch einmal in die Hand genommen wird.

Besonders interessant, weil mit persönlicher Note, sind Flyer und Handzettel, die die Praxis extra für ihre Kunden anfertigt. Die sind dann natürlich mit dem Praxislogo versehen, haben eine Zeile für den Tiernamen und können auch so gestaltet sein, dass sie nur die für das Tier wichtigen Informationen enthalten und nicht einen Querschnitt aus allen möglichen Infos. Das hat den Vorteil, dass der Tierhalter sehr persönliche und genau auf seinen Fall zugeschnittene, d. h. quasi maßgeschneiderte Fakten und Unterstützung enthält, was den Wert des Materials erhöht. Denkbar sind dabei eigens von der Praxis erstellte und in der EDV hinterlegte Vorlagen für verschiedene Situationen, die dann je nach Fall mit den persönlichen Empfehlungen und Verschreibungen für das einzelne Tier bearbeitet, ausgedruckt und abgegeben werden. Das kostet nicht viel Zeit, macht enormen Eindruck auf den Kunden und hilft, dass die Informationen auch bei den 
(meist vorhandenen) anderen Familienmitgliedern auch ankommen.

Spezialpraxen benötigen für die Kundenansprache jeweils zu ihren besonderen Leistungen passende Materialien und darüber hinaus Materialien, mit denen sie mit den überweisenden Kollegen kommunizieren - denn das sind ja die eigentlichen „Endkunden“.

\section{TIERÄRZTLICHE INFOQUELLEN FÜR \\ DEN TIERHALTER}

- attraktive Homepage mit wechselnden Schwerpunktthemen

- Website-Bereiche mit Informationen zu besonderen Themen

- Downloads auf der Homepage für übersichtliche und kurze Inhalte zu speziellen Gesundheitsfragen

- Links zu weiteren Seiten mit geprüften, vertrauenswürdigen Fakten

- personalisierte Handzettel und Broschüren zur Abgabe nach der Behandlung

\section{Info-Quellen}

Zusätzlich sollte die Praxishomepage attraktiv und mit vielen Informationen zu verschiedenen Themen "gespickt" sein ( $\triangleright$ Abb. 3). Das bedeutet jetzt nicht, dass Kunden seitenlange Abhandlungen lesen möchten - sie möchten Fakten kurz, übersichtlich, prägnant und mit TO DOs erhalten, ohne allzu sehr suchen zu müssen. Dazu ist es sinnvoll, diese Infos in einem bestimmten Seitenbereich auch zum Download anzulegen. In der Menüleiste der Hauptseite kann z. B. ein Begriff wie „Infothek“ oder ähnliches einladen, diesen Bereich zu erforschen. Dort mit einem Klick angekommen, gibt es die Bereiche „Katze“, „Hund“, „Meerschwein“ etc., von denen der User dann zu den einzelnen Dauerbrenner-Themen wie z. B. Ernährung, Parasiten, Kastration usw. kommt.

Ergänzend können wechselnde, aktuelle und evtl. brisante Themen wie z.B. Bernsteinkette oder Kokosöl als „Teaser“ (beschrifteter Button zum Anklicken) auch auf der Startseite zum Lesen animieren und direkt mit 1 Klick zum Thema leiten. Wichtig dabei ist, dass die Homepage gepflegt wird, d.h. sich immer wieder mit Informationen und News erneuert und nicht (wie leider öfter zu sehen) in der Rubrik „Aktuelles“ als erste noch „Neuigkeiten“ von 2016 stehen.

Für Kunden immer auch interessant sind Links zu weiteren Informationsquellen, die die Praxis natürlich auch regelmäßig prüfen (ob sie zur Praxisphilosophie passen und funktionieren) und auffrischen sollte. Diese Links können auch je nach Fall in die von der Praxis ausgehändigten Ma-

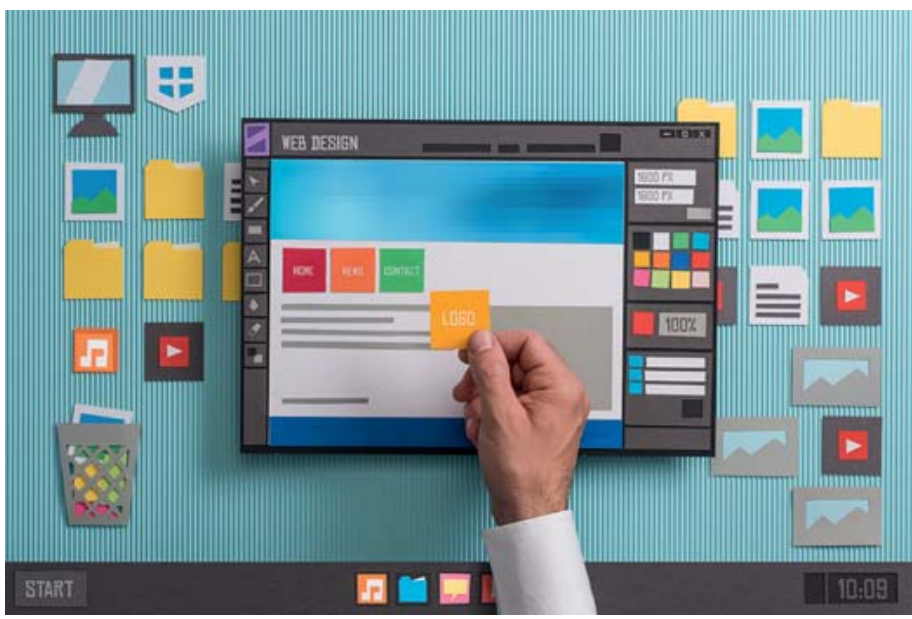

- Abb. 3 Eine interessante Homepage mit aktuellen und wechselnden Info-Komponenten zu verschiedenen Themenbereichen bindet Kunden und hilft, die eigene Website ins Zentrum der Tierhalter-Recherchen zu positionieren. @ stokkete - stock.adobe.com

terialien integriert werden - so hat der Kunde noch mehr Auswahl an Themen und Seiten, die die Praxismeinung unterstützen und unterstreichen.

\section{Fazit}

Mit einer optimierten Zeitplanung und einer guten Kommunikationsstrategie kann es der Praxis gelingen, die Nummer 1 der Informationsquellen für den Kunden zu werden und den Kollegen „Google“ etwas an den Rand zu drängen. Entscheidend dabei ist, dass die Praxis großzügig und proaktiv an den Kunden herantritt und Informationen liefert. Dann ist das Team „am Drücker“ und nicht externe Meinungsmacher.

Und natürlich gibt es noch mehr und digitale Kommunikationsmöglichkeiten, doch es gilt, zunächst die hier aufgezeigten gut und aktuell zu bedienen, bevor Facebook, Instagram, YouTube \& Co bearbeitet werden - die persönliche Kommunikation und eine aktuelle, im Netz gut sichtbare Homepage sind immer noch (zunächst) das Zentrum der Kundenkommunikation.

\section{Korrespondenzadresse}

\section{Antje Blättner}

Tierärztin und Kommunikationstrainerin a.blaettner@vetkom.de

\section{Bibliografie}

DOI https://doi.org/10.1055/a-0713-7596

Veterinärspiegel 2018; 28: 171-175

(c) Georg Thieme Verlag KG Stuttgart · New York

ISSN 0940-8711 